\title{
Respuestas cerebrales al silencio: hacia una nueva forma de evaluación clínica de la audición
}

\author{
Brain responses to silence: towards a new clinical evaluation of audition
}

\author{
Pablo Henríquez C. ${ }^{1,2,5}$, Paul Délano R. ${ }^{1,2,3,4}$
}

\section{Resumen}

Nuestro cerebro tiene diferentes estados de funcionamiento, uno de ellos es la ausencia de estimulación auditiva o estado de silencio. Para evaluar la capacidad de detección de períodos de silencio auditivo se pueden utilizar diversas pruebas, incluyendo evaluaciones conductuales y electrofisiológicas. En esta última línea existen numerosos estudios que han caracterizado la respuesta cerebral al silencio mediante oscilaciones cerebrales y potenciales evocados con silencio. Esta área promete una importante aplicabilidad clínica, por ejemplo, en patologías como tinnitus, condición en la que no existe el estado de silencio, en donde se cree que la evaluación de respuestas cerebrales al silencio podría surgir como un nuevo marcador o elemento diagnóstico. En este artículo se presenta una revisión de los distintos métodos utilizados para evaluar respuestas cerebrales al silencio en modelos animales y en humanos.

Palabras clave: silencio, gap in noise, tinnitus, potenciales evocados, oscilaciones cerebrales.

\begin{abstract}
The brain has different states of operation, one of them is the absence of auditory stimulation or silent states. To assess the capacity to detect auditory silent periods, various tests have been developed, including behavioral and electrophysiological evaluations. Brain responses to silence can be evaluated with brain oscillations and evoked potentials. This area promises important clinical applicability, for example, in pathologies like tinnitus, a condition in which there is no silent state, where it is believed that the evaluation of brain responses to silence could emerge as a new marker or diagnostic element. In this article we review different methods used for assessing brain responses to sound in animal models and humans.
\end{abstract}

Keywords: silence, gap in noise, tinnitus, auditory evoked potentials, brain oscillations.
'Departamento de Otorrinolaringología, Hospital Clínico de la Universidad de Chile. Santiago, Chile.

${ }^{2}$ Laboratorio de Neurobiología de la Audición, Departamento de Neurociencia, Facultad de Medicina, Universidad de Chile. Santiago, Chile.

${ }^{3}$ Biomedical Neuroscience Institute, BNI, Facultad de Medicina, Universidad de Chile. Santiago, Chile.

${ }^{4}$ Centro Avanzado de Ingeniería Eléctrica y Electrónica, AC3E, Universidad Técnica Federico Santa María. Valparaíso, Chile. ${ }^{5}$ Programa Doctorado en Ciencias Médicas, Escuela de Postgrado, Facultad de Medicina, Universidad de Chile. Santiago, Chile.

Los autores declaran no tener conflictos de interés. PDR es financiado por ANID BASAL FB008, Proyecto ICN09_015, Vicerrectoría de Investigación y Desarrollo (VID) de la Universidad de Chile, código proyecto: ENL 19/20 y Fundación Guillermo Puelma.

Recibido el 19 de octubre de 2020. Aceptado el 5 de diciembre de 2020 .

Correspondencia:

Paul Délano R.

Santos Dumont 999

Departamento de Otorrinolaringología Hospital Clínico de la Universidad de Chile. Santiago, Chile.

Email: pdelano@med.uchile.cl

\section{Introducción}

Nuestro cerebro funciona en diferentes estados como, por ejemplo, el sueño y la vigilia ${ }^{1}$. En el caso del sistema auditivo, se pueden distinguir los estados cerebrales de estimulación auditiva y silencio. Si bien, la mayoría de la investigación en audición se centra en el estudio de cómo el sistema auditivo responde a los estímulos acústicos (clics, tonos puros, fonemas, palabras), las estructuras de la vía auditiva pueden responder al silencio. Uno de los exámenes perceptuales y electrofisiológicos más conocidos de respuesta al silencio es la prueba de "gap in noise", que se utiliza para evaluar el procesamiento temporal au- 
ditivo $^{2}$. Sin embargo, existen otros tipos de respuestas al silencio como, por ejemplo, las oscilaciones cerebrales, que pueden medirse en un electroencefalograma (EEG). En este artículo se presenta una visión panorámica de las respuestas cerebrales al silencio y de cómo estas, a futuro, podrían ser utilizadas en la práctica clínica, por ejemplo, en patologías como tinnitus, condición en la que no existe el estado de silencio, o en el estudio de pacientes con sordera profunda e implantes cocleares, quienes antes de ser operados sólo conocían el estado de silencio.

\section{Respuestas perceptuales al silencio}

El estudio de los mecanismos neurobiológicos del procesamiento auditivo central incluye características espectrales, temporales e interhemisféricos de la señal acústica, cuyos primeros estudios datan de Garner en $1947^{3}$. De estos elementos, el procesamiento temporal es considerado uno de los componentes más importantes, ya que nos permite procesar y percibir adecuadamente todos los elementos del tiempo en la audición y, de esta manera, distinguir en forma precisa un momento de silencio dentro de una secuencia de estímulos auditivos.

La estructura acústica de fonemas y palabras presenta múltiples segmentos de silencio de corta duración (gaps de silencio, del orden de los milisegundos; Figura 1) que son esenciales para la inteligibilidad de las palabras ${ }^{4}$. De esta forma, los gaps de silencio entre estímulos auditivos permiten una adecuada discriminación temporal auditiva, es decir, diferenciar dos estímulos sonoros como distintos ${ }^{2,5,6}$. La evaluación perceptual de las respuestas a gaps de silencio para estimar la discriminación temporal auditiva se viene realizando hace varias décadas ${ }^{3,7,9}$, siendo el método más utilizado y estandarizado la prueba de gaps in noise (GIN), desarrollada por Musiek y colaboradores en el año $2005^{10}$. La evaluación de umbrales de GIN permite determinar si una persona tiene habilidades de percepción temporal auditiva en rango normal, que para GIN debe ser de 2 a 3 milisegundos de silencio ${ }^{11}$. Por otro lado, se han descrito otros tipos de pruebas de gaps de silencio en estímulos acústicos, como de tonos puros (gap in tone), o de dos tonos ( $\mathrm{fl} y$ f2) que provocan una emisión otoacústica por producto de distorsión (gap in DPOAE) ${ }^{12}$, los cuales se utilizan en investigación audiológica. De manera importante, una alteración en la detección de gaps de silencio se correlaciona con alteraciones cognitivas, en lectoescritura y aprendizaje en la infancia ${ }^{13}$, así como también en alteraciones del procesamiento auditivo central en presbiacusia ${ }^{14}$.

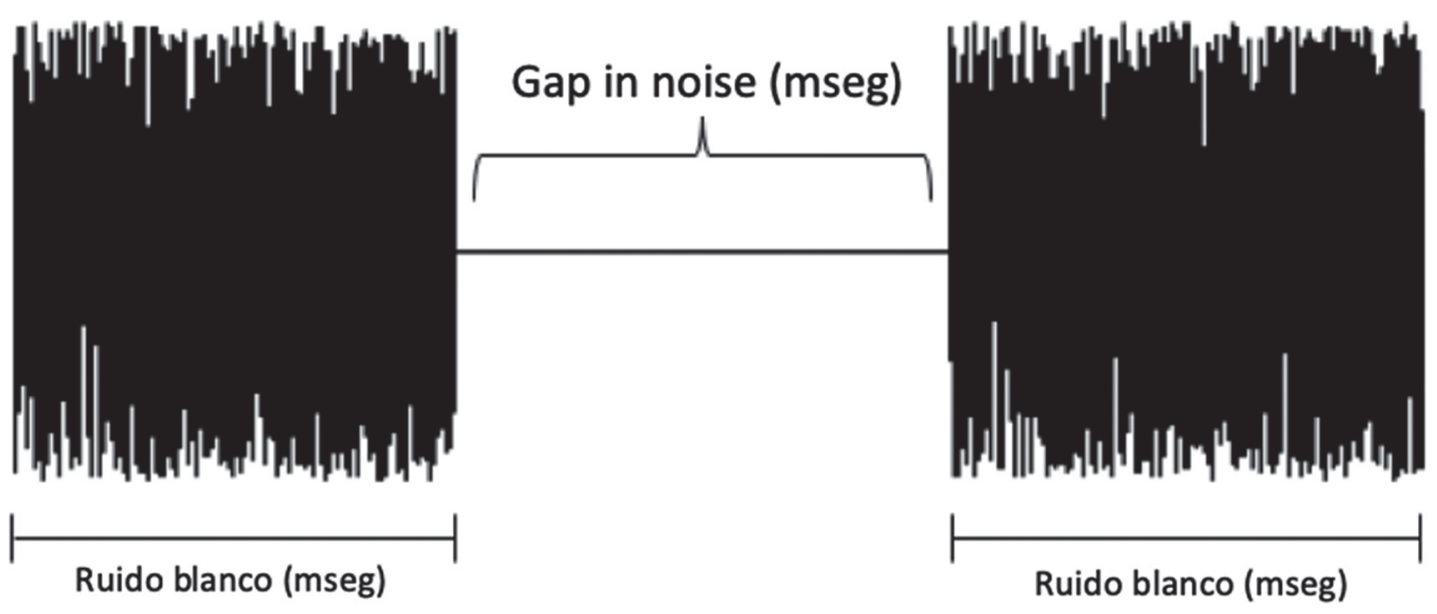

Figura 1. Oscilograma acústico de gap in noise. La imagen muestra un ejemplo ilustrativo de un oscilograma del estímulo acústico utilizado en las pruebas de gap in noise. El registro ilustra un estímulo tipo ruido blanco, el cual es abruptamente interrumpido por un período de silencio (gap in noise) de duración variable en el rango de milisegundos. 
Los umbrales de GIN pueden evaluarse bajo dos condiciones ${ }^{2}$ : La primera de ellas se conoce como evaluación de gaps "dentro del canal", que consiste en evaluar la detección del silencio entre dos estímulos auditivos con similar composición espectral ${ }^{15}$; la segunda se conoce como evaluación de gaps "a través del canal”, la cual evalúa la detección del gap entre dos estímulos con composición espectral diferente ${ }^{16}$. Entre ambas pruebas, la primera presenta umbrales de detección menores, ya que el gap sólo es interpretado como una interrupción de la señal sonora. La prueba "a través del canal", por otro lado, implica una detección del término de la primera señal y del comienzo de la segunda, y una comparación entre ambos grupos neuronales estimulados, por lo que son más los recursos neurales implicados, generando que la tarea sea más demandante cognitivamente y sus valores también sean mayores $^{17}$. Los umbrales de GIN se han estudiado también en la escucha eléctrica con implantes cocleares. En estos pacientes se ha visto que, al igual que en la escucha acústica, los umbrales de GIN aumentan al presentarse estímulos auditivos "entre canales", al fluctuar más la amplitud de la envolvente del sonido, o bien al presentar un sonido de igual composición espectral, pero de manera dicótica, es decir, el primer estímulo en un oído, y el segundo en el contralateral ${ }^{18,19}$.

\section{Potenciales evocados auditivos al silencio}

Los potenciales evocados al silencio pueden ser medidos mediante electrodos de superficie, y podemos caracterizar distintos niveles de medición a lo largo de la vía auditiva. Sabemos que en el nervio auditivo las fibras nerviosas presentan una tasa de descarga basal en ausencia de estimulación auditiva ${ }^{20}$. Si bien, no se han descrito potenciales evocados por silencio a nivel coclear, se puede medir el ruido eléctrico de la ventana redonda o del tímpano en condiciones de silencio ${ }^{21,22}$. Esta señal bioeléctrica fue descrita en 1990 por Dolan y cols. en modelos animales, y corresponde a la actividad espontánea del nervio auditivo en condiciones de silencio $^{23}$. Este tipo de potencial aún no se utiliza en la práctica clínica, pero podría ser de utilidad para evaluar la función del nervio auditivo en pacientes candidatos a implante coclear o con tinnitus. Por otro lado, existe evidencia de que el silencio puede afectar la duración y la morfología de los potenciales evocados auditivos de tronco encefálico, donde se ha reportado que gaps de duración más cortas se asocian a una disminución en la amplitud en las ondas de potenciales evocados de latencia media ${ }^{21,22}$.

Así como clics, tonos u otros estímulos auditivos pueden generar potenciales evocados en la corteza auditiva, el inicio de un período de silencio ( $g a p s$ de silencio) también provoca potenciales evocados corticales ${ }^{24}$, que se pueden medir por EEG. Los potenciales evocados corticales por gaps de silencio se caracterizan por la presencia de dos deflexiones positivas (P1 y P2) interrumpidos por una deflexión negativa (N1) (Figura 2). Estos complejos se generan tanto en corteza auditiva primaria como secundaria, e involucran a numerosas estructuras, como hipocampo, corteza temporal lateral, áreas neocorticales y sistema reticular ${ }^{25}$, y nos permiten inferir eventos cognitivos implicados en tareas propuestas ${ }^{26}$.

Cuando se presentan gaps de silencio con una duración mayor al umbral de detección de cada sujeto, estos gatillarán potenciales evocados corticales que tendrán una latencia de P1 de 50 a 80 ms, N1 de 60 a 160 ms y P2 de 180 a $200 \mathrm{~ms}^{24,27-30}$. El hecho de que las respuestas se obtengan en relación con el inicio del período de silencio implica que la persona respondió al término de la estimulación auditiva y, por ende, que el individuo examinado detectaba el estímulo auditivo que precede al silencio. De esta forma la presencia de un potencial evocado a gaps de silencio es una forma indirecta de determinar audición a un estímulo auditivo de intensidad y frecuencia específica (el que se encuentra justo antes de empezar el silencio), por lo que podría ser utilizada como medida en audiología clínica.

El potencial evocado cortical a gaps de silencio se modula por diversos estados cognitivos. El componente P1 juega un rol importante con la orientación de la atención, y su mayor latencia se asocia a peor rendimiento en tareas de atención y memoria ${ }^{31}$. Por otro lado, la amplitud de N2 se asocian con mejor rendimiento en tareas de atención sostenida ${ }^{24}$. En esta misma línea, Harris y cols. en 2012 evidenciaron que al comparar la detección de potenciales evocados a GIN y rendimiento en 
tareas cognitivas y de velocidad psicomotriz entre personas jóvenes y adultos mayores, los adultos mayores tienen peor rendimiento que jóvenes en detectar los gaps tanto en escucha activa (detectar dirigidamente el gap de silencio) como pasiva (detectar el gap mientras se realiza otra tarea). Existiendo, además, una correlación positiva entre peor detección de gaps y peor rendimiento cognitivo y/o psicomotriz ${ }^{24}$. De esta forma, podemos hacernos una idea de la importancia que tiene el procesamiento auditivo cerebral, evaluado en función de la detección de gaps, como una medida del procesamiento cognitivo subyacente. Hallazgos similares se encontraron en el estudio de Lister y cols., 2011, en donde los adultos mayores, en comparación a sujetos jóvenes, exhibieron mayores latencias de P2, sugiriendo un enlentecimiento de la transmisión neural y de la capacidad inhibitoria de estímulos irrelevantes en la población envejecida ${ }^{28}$.

\section{Oscilaciones cerebrales en respuesta al silencio}

Además de los potenciales evocados auditivos, se puede estudiar la actividad cerebral espontánea o inducida por estímulos a través de la medición de las oscilaciones cerebrales. Clásicamente se describen las oscilaciones en el EEG en el rango de $1-30 \mathrm{~Hz}$, que se pueden agrupar en bandas de frecuencias: ondas alfa $(9-12 \mathrm{~Hz})$, beta $(13-20 \mathrm{~Hz})$, gamma $(>20 \mathrm{~Hz})$, theta $(4-8 \mathrm{~Hz})$ y delta $(1-3 \mathrm{~Hz})^{32}$. Las oscilaciones que predominan en el EEG dependen de los distintos estados cerebrales como, por ejemplo, el sueño y la vigilia, y en el caso del sistema auditivo también se observan diferentes patrones en las oscilaciones cerebrales, dependiendo de los estados de estimulación auditiva o silencio.

Estudios en modelos de roedores (ratas y chinchillas) han mostrado la presencia de oscilaciones de baja frecuencia en la corteza auditiva (bandas theta, alfa y beta $[<20 \mathrm{~Hz}]$ ), que aparecen luego de periodos de silencio y que disminuyen la amplitud de las respuestas a estímulos auditivos ${ }^{33-35}$ (Figura 3). Este tipo de respuestas cerebrales amplifica la primera respuesta a un estímulo auditivo que rompe el estado de silencio. Se cree que esto podría tener una explicación evolutiva, ya que usualmente en la naturaleza los sonidos que rompen periodos de silencio prolongados pueden ser una señal de alerta importante.

En el año 2017, Yusuf y colaboradores mostraron una reducción de las oscilaciones inducidas de baja frecuencia $(<20 \mathrm{~Hz})$ en gatos con sordera profunda congénita bilateral con implantes cocleares ${ }^{36}$. Estos resultados muestran que la actividad oscilatoria inducida por periodos de silencio de la corteza auditiva pue-
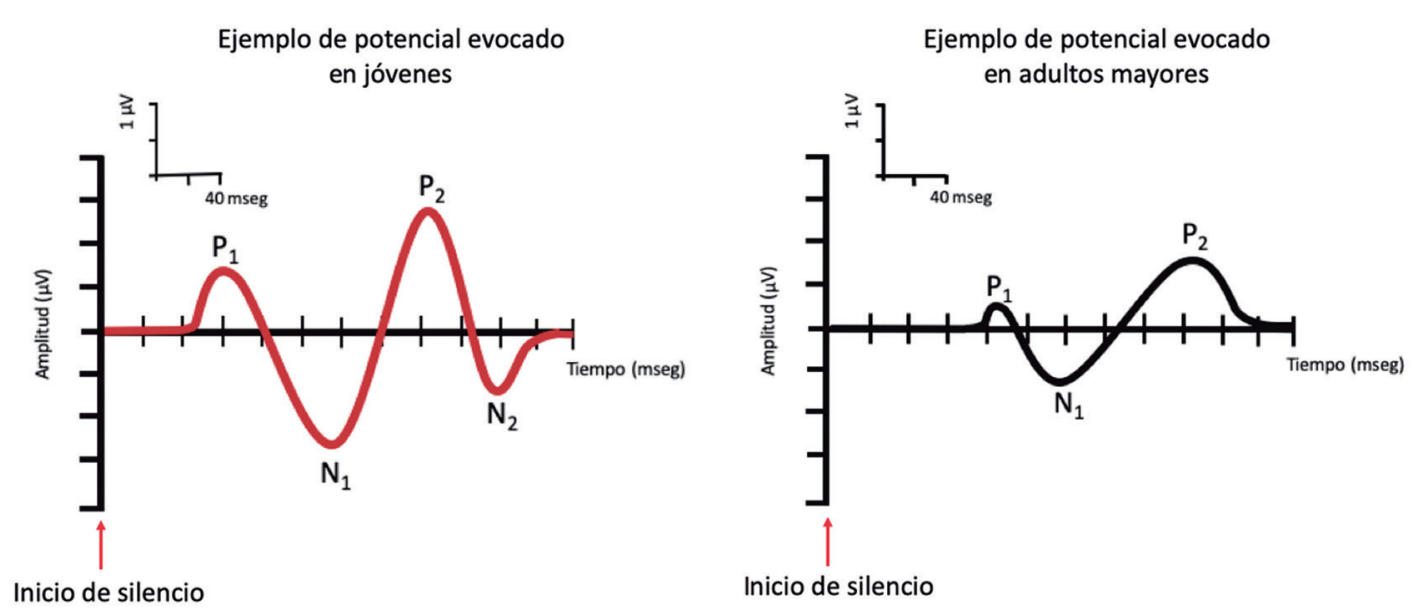

Figura 2. Potenciales evocados corticales a gaps de silencio. Se ilustran dos ejemplos de potenciales evocados a gaps de silencio en jóvenes (a la izquierda) y adultos mayores (a la derecha). Se describen característicamente dos deflexiones positivas $\left(P_{1}\right.$ y $P_{2}$ ) y dos deflexiones negativas $\left(N_{1}\right.$ y $\left.N_{2}\right)$. Nótese que en adultos mayores hay una disminución de amplitud en todas las deflexiones, así como un $N_{2}$ prácticamente inexistente. 
de ser utilizada como un índice del nivel de audición en implantes cocleares. Sin embargo, el campo de las oscilaciones cerebrales inducidas por silencio es aún un terreno inexplorado en seres humanos, que puede tener aplicaciones clínicas importantes en la audiología clínica.

\section{Tinnitus y respuestas al silencio}

La fisiopatología del tinnitus propone que el trauma acústico provoca una "de-aferentación" del nervio auditivo, y en forma compensatoria una hiperactivación en las vías auditivas centrales. Cuando el tinnitus persiste de forma crónica se alteran, además, redes de procesamiento cerebral de emociones $\mathrm{y}$ funciones cognitivas que perpetúan el cua$\mathrm{dro}^{37}$. La presencia de tinnitus crónico impide a una persona estar en el estado de silencio, y de hecho en situaciones silenciosas, con poco ruido de fondo, los pacientes suelen percibir el tinnitus de mayor intensidad ${ }^{38}$. De esta forma, el tinnitus impide que el cerebro ingrese al estado de silencio, lo que sería similar al caso de pacientes con insomnio que no pueden entrar al estado cerebral del sueño. Las consecuencias fisiopatológicas de la ausencia de estado de silencio en pacientes que sufren de tinnitus aún son materia de estudio.

La incapacidad de percibir silencio en pacientes con tinnitus puede medirse a través de pruebas conductuales en modelos animales y en humanos como, por ejemplo, la percepción de GIN. Una de las conductas más estudiadas en roedores es el reflejo acústico de sobresalto (acoustic startle reflex, ASR, por sus siglas en inglés), la cual es una respuesta conductual motora a sonidos bruscos de alta intensidad (sonido de sobresalto), cuyo correlato neural se encuentra en circuitos del tronco cerebral ${ }^{39}$. El ASR puede ser suprimido al presentar un estímulo acústico de baja intensidad o un gap de silencio antes del sonido de sobresalto, fenómeno conocido como "inhibición prepulso del ASR" 39,40 . Estudios en roedores expuestos a trauma acústico para inducir tinnitus muestran que los animales que desarrollan tinnitus carecen de la inhibición prepulso que provoca el gap de silencio en el ASR ${ }^{41,42}$ (Figura 4). De esta forma, de manera indirecta, se puede asumir que el animal percibe un tinnitus que le impide detectar los gaps de silencio ${ }^{30}$.

En humanos se han realizado estudios

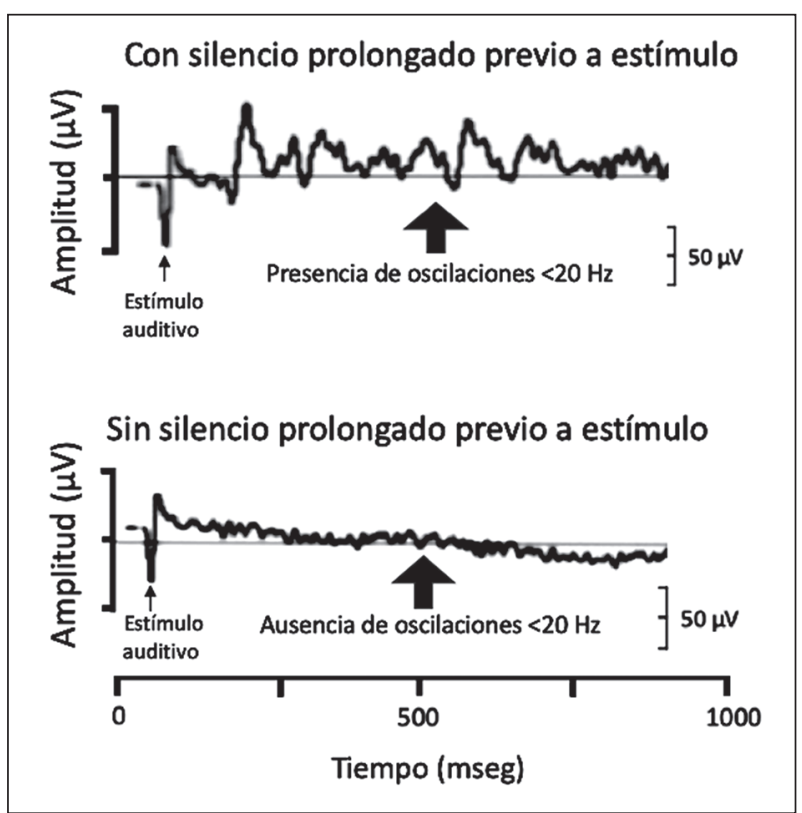

Figura 3. Oscilaciones cerebrales inducidas por silencio. Frente a un periodo prolongado de silencio (> 5 segundos), en la corteza auditiva de chinchillas, se registran oscilaciones de baja frecuencia $(<20 \mathrm{~Hz}$, flecha) posterior al potencial evocado cortical con clic (imagen superior). Estímulos auditivos no precedidos de silencios prolongados provocan respuestas evocadas en la corteza auditiva sin oscilaciones posteriores (imagen inferior). Figura modificada de Délano y cols., $2008^{33}$.

conductuales similares, pero con resultados contradictorios, encontrando en algunos casos alteraciones en la detección de GIN en sujetos con tinnitus ${ }^{43}$, y en otros no $^{44,45}$. Por ejemplo, Campolo y colaboradores en el año 2013 utilizaron gaps de $50 \mathrm{~ms}$ para evaluar si estos suprimían o no el ASR en humanos, y encontraron que en sujetos con tinnitus se perdía la inhibición prepulso, pero no la detección del gap auditivo ${ }^{45}$. Boyen y colaboradores se plantearon la duda si acaso los resultados anteriores pudiesen ser explicados por ser el gap de $50 \mathrm{~ms}$ una tarea sencilla y poco desafiante cognitivamente, e intentaron resolverla realizando una tarea conductual adaptativa en donde se le entregaba a sujetos un sonido de similar tonalidad a su tinnitus, pero con duraciones progresivamente menores de gaps, de manera de hacer esta tarea más desafiante. En sus resultados no encontraron diferencias significativas en la detección de gaps respecto al grupo control, tanto en duración del gap como en contenido frecuencial ${ }^{44}$. Estos ejem- 

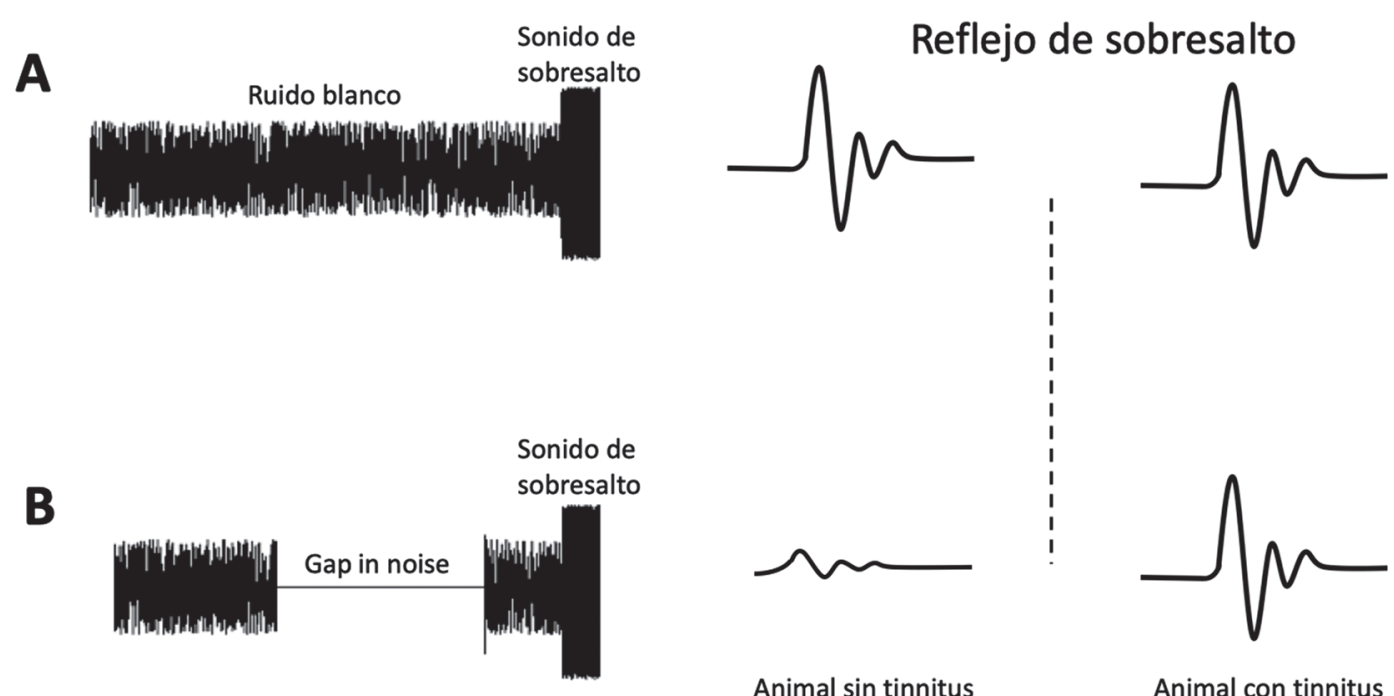

Figura 4. Respuesta de sobresalto con gaps de silencio en modelos animales de tinnitus. Se muestran dos condiciones: A. Reflejo de sobresalto con ruido blanco de fondo, seguido de un sonido de alta intensidad que desata el reflejo acústico de sobresalto en roedores. B. Reflejo de sobresalto con un gap de silencio antes del sonido. Al evaluar las respuestas en el reflejo acústico de sobresalto es posible ver que en condiciones normales (animal sin tinnitus) el reflejo es inhibido cuando se antepone un gap al sonido de exaltación, sin embargo, en el modelo de tinnitus el reflejo no se inhibe (porque el tinnitus "llena" el espacio de silencio, impidiendo la percepción de silencio antes del sonido de sobresalto), registrándose respuestas similares a la situación sin gap. Figura modificada de Paul y cols., $2018^{30}$.

plos plantean una razonable duda respecto a cómo el tinnitus podría afectar la resolución temporal alterando el ASR, pero no la detección de gaps, lo cual podría en parte explicarse por estructuras neurales distintas implicadas: En el caso del ASR, estructuras subcorticales como en núcleo reticular pontino caudal y neuronas del nervio auditivo ${ }^{46}$; mientras que en las tareas psicoacústicas se involucran predominantemente estructuras corticales ${ }^{44}$. Esta variedad de resultados distintos y la falta de conocimiento fisiopatológico de los mecanismos implicados en tinnitus, cuestionan la objetividad del uso del GIN en humanos para el estudio del tinnitus en pruebas psicoacústicas.

$\mathrm{Al}$ revisar otras técnicas como la electroencefalografía, Brandon y colaboradores (2018) demostraron que en sujetos normo oyentes y sin tinnitus, al medir potenciales evocados corticales en respuesta a gaps de silencio de $20 \mathrm{~ms}$, es posible registrar valores de $\mathrm{N} 1$ con latencias y amplitudes estables con baja variabilidad intersujeto. Esto permitiría establecer comparaciones entre sujetos con y sin tinnitus, en donde se podrían objetivar diferencias en potenciales corticales dados por el efecto "enmascarador de silencio" que generaría el tinnitus ${ }^{30}$. Por otro lado, recientemente, Morse y colaboradores (2019) encontraron un aumento en la latencia de P1 al comparar potenciales evocados corticales a GIN entre un grupo con tinnitus y otro control ${ }^{47}$. Como podemos ver, el estudio de las respuestas al silencio aplicado a tinnitus es un campo aún en desarrollo, que probablemente en un futuro cercano sea de utilidad en audiología clínica.

\section{Conclusión}

El cerebro en el estado de silencio tiene características electrofisiológicas y conductuales que pueden ser medidas con potenciales evocados, oscilaciones cerebrales y tareas perceptuales. El estudio de las respuestas al silencio en el sistema auditivo es un campo abierto para la investigación clínica en hipoacusia, implantes cocleares y tinnitus. 


\section{Bibliografía}

1. Hobson JA, Pace-Schott EF. The cognitive neuroscience of sleep: Neuronal systems, consciousness and learning. Nat Rev Neurosci. 2002;3:679-693. doi: 10.1038/nrn915.

2. Marcotti FA, Alvear VB. Pruebas de fusión auditiva y de detección de gaps: Evaluación de la resolución auditiva temporal. Rev Otorrinolaringol Cir Cabeza Cuello. 2019;79:248-260. doi: 10.4067/s071848162019000200248 .

3. Garner WR. The Effect of Frequency Spectrum on Temporal Integration of Energy in the Ear. J Acoust Soc Am. 1947;19(5):808-815. doi: 10.1121/1.1916625.

4. Elangovan S, Stuart A. Natural boundaries in gap detection are related to categorical perception of stop consonants. Ear Hear. 2008;29:761-774. doi: 10.1097/ AUD.0b013e318185ddd2.

5. Green DM. Temporal auditory acuity. Psychol Rev. 1971;78:540-551. doi: 10.1037/h0031798.

6. Anderson LA, Linden JF. Mind the gap: Two dissociable mechanisms of temporal processing in the auditory system. J Neurosci. 2016;36:1977-1995. doi: 10.1523/JNEUROSCI.1652-15.2016.

7. Miller GA, Taylor WG. The Perception of Repeated Bursts of Noise. J Acoust Soc Am. 1948;20(2):171-182. doi: 10.1121/1.1906360.

8. Hirsh IJ. Auditory Perception of Temporal Order. J Acoust Soc Am. 1959 Jun 11;31(6):759-767. doi: 10.1121/1.1907782.

9. Plomp R. Rate of Decay of Auditory Sensation. J Acoust Soc Am. 1964;36(2):277-282. doi: 10.1121/1.1918946.

10. Musiek FE, Shinn JB, Jirsa R, Bamiou DE, Baran JA, Zaida E. GIN (Gaps-In-Noise) test performance in subjects with confirmed central auditory nervous system involvement. Ear Hear. 2005;26:608-618. doi: 10.1097/01.aud.0000188069.80699.41.

11. Phillips DP. Auditory Gap Detection, Perceptual Channels, and Temporal Resolution in Speech Perception. J Am Acad Audiol. 1999;10:343-354.

12. Dragicevic CD, Marcenaro B, Navarrete M, Robles L, Delano PH. Oscillatory infrasonic modulation of the cochlear amplifier by selective attention. PLoS One. 2019;14(1):e0208939. doi:10.1371/journal. pone. 0208939 .

13. McCroskey RL, Kidder HC. Auditory fusion among learning disabled, reading disabled, and normal children. J Learn Disabil, 1980;13:69-76. doi:10.1177/002221948001300205.

14. Torrente M, Leiva A. Evaluación de procesamiento auditivo y percepción sonora en sujetos con presbiacusia. Rev Otorrinolaringol Cir Cabeza Cuello. 2018;78(4):363-368. doi:10.4067/s071775262018000400363 .

15. Purcell DW, John SM, Schneider BA, Picton TW. Human temporal auditory acuity as assessed by envelope following responses. J Acoust Soc Am. 2004; 116(6):3581-3593. doi: 10.1121/1.1798354.

16. Grose JH, Hall JW, Buss E, Hatch D. Gap detection for similar and dissimilar gap markers. J Acoust Soc Am. 1999;106(4):2206-2206. doi: 10.1121/1.1354983.

17. Formby C, Gerber MJ, Sherlock LP, Magder LS. Evidence for an across-frequency, between-channel process in asymptotic monaural temporal gap detection. J Acoust Soc Am. 1998;103(6):3554-3560. doi: $10.1121 / 1.423084$

18. Grose JH, Buss E. Within- and across-channel gap detection in cochlear implant listeners. J Acoust Soc Am. 2007;122(6):3651-3658. doi: 10.1121/1.2793608.

19. Garadat SN, Pfingst BE. Relationship between gap detection thresholds and loudness in cochlearimplant users. Hear Res. 2011;275(1-2):130-138. doi: 10.1016/j.heares.2010.12.011.

20. Legatt AD. Electrophysiologic auditory tests. En Handbook of Clinical Neurology. Elsevier. 2015:289311.

21. Pardo-Jadue J, Dragicevic CD, Bowen M, Delano PH. On the Origin of the $1,000 \mathrm{~Hz}$ Peak in the Spectrum of the Human Tympanic Electrical Noise. Front Neurosci 2017;11:395. doi:10.3389/fnins.2017.00395.

22. Pardo J, Dragicevic C, Bowen M, Délano P. Ruido eléctrico de la ventana redonda. Rev Otorrinolaringol Cir Cabeza Cuello. 2015;75(2):173-178. doi:10.4067/ S0718-48162015000200014.

23. Dolan DF, Nuttall AL, Avinash G. Asynchronous neural activity recorded from the round window. J Acoust Soc Am. 1990;87:2621-2627. doi:10.1121/1.399054.

24. Harris KC, Wilson S, Eckert MA, Dubno JR. Human evoked cortical activity to silent gaps in noise: Effects of age, attention, and cortical processing speed. Ear Hear. 2012;33:330-339. doi: 10.1097/ AUD.0b013e31823fb585.

25. Tremblay, K.\& Clinard C. Cortical Auditory-Evoked Potentials. In: J Katz, M Chasin, K English, L Hood \& K Tillery, Handbook of Clinical Audiology. Wolters Kl. Philadelphia; 2015. p. 249-266.

26. Barajas de Praat, J., Zenker F\& FR. Potenciales evocados auditivos. In: C Suárez \& L Carcedo Tratado de otorrinolaringología y cirugía de cabeza y cuello. Ed. Paname. 2007. p. 1133-1155.

27. Lister JJ, Maxfield ND, Pitt GJ, Lister J. Cortical Evoked Response to Gaps in Noise: WithinChannel and Across-Channel Conditions. Ear Hear. 2007;28:862-878. doi: 10.1097/ AUD.0b013e3181576cba.

28. Lister JJ, Maxfield ND, Pitt GJ, González VB. Auditory evoked response to gaps in noise: Older adults. Int J Audiol. 2011;50(4):211-225. doi: 10.3109/14992027.2010.526967.

29. Palmer SB, Musiek FE. N1-P2 recordings to gaps in broadband noise. J Am Acad Audiol. 2013;24:37-45. doi: 10.3766/jaaa.24.1.5. 


\section{ARTÍCULO DE REVISIÓN}

30. Paul BT, Schoenwiesner M, Hébert S. Towards an objective test of chronic tinnitus: Properties of auditory cortical potentials evoked by silent gaps in tinnitus-like sounds. Hear Res. 2018;366:90-98. doi: 10.1016/j.heares.2018.04.005.

31. Čeponiene R, Alku P, Westerfield M, Torki M, Townsend J. ERPs differentiate syllable and nonphonetic sound processing in children and adults. Psychophysiology. 2005;42(4):391-406. doi: 10.1111/j.1469-8986.2005.00305.x.

32. Buzsáki G, Draguhn A. Neuronal oscillations in cortical networks. Science. 2004;304:1926-1929.

33. Delano PH, Pavez E, Robles L, Maldonado PE. Stimulus-dependent oscillations and evoked potentials in chinchilla auditory cortex. J Comp Physiol A Neuroethol Sens Neural Behav Physiol. 2008;194(8):693-700. doi:10.1007/s00359-008-03404.

34. Cotillon N, Nafati M, Edeline JM. Characteristics of reliable tone-evoked oscillations in the rat thalamocortical auditory system. Hear Res. 2000;142:113-30. doi: 10.1016/s0378-5955(00)00016-2.

35. Cotillon-Williams N, Edeline JM. Evoked oscillations in the thalamo-cortical auditory system are present in anesthetized but not in unanesthetized rats. $J$ Neurophysiol. 2003;89(4):1968-1984. doi: 10.1152/ jn.00728.2002.

36. Yusuf PA, Hubka P, Tillein J, Kral A. Induced cortical responses require developmental sensory experience. Brain. 2017;140(12):3153-3165. doi: 10.1093/brain/ awx286.

37. Wimmer J, Donoso R, Leiva A, Breinbauer $\mathrm{H}$, Délano P. Tinnitus: Una patología cerebral. Rev Otorrinolaringol Cirugía Cabeza Cuello 2019;79:125136. doi: $10.4067 /$ S0718-48162019000100125.

38. Roberts LE, Eggermont JJ, Caspary DM, Shore SE, Melcher JR, Kaltenbach JA. Ringing ears: The neuroscience of tinnitus. J Neurosci. 2010;30:1497214979. doi: .10.1523/JNEUROSCI.4028-10.2010.

39. Basavaraj S, Yan J. Prepulse Inhibition of Acoustic
Startle Reflex as a Function of the Frequency Difference between Prepulse and Background Sounds in Mice. PLoS One. 2012;7(9). doi: 10.1371/journal. pone. 0045123 .

40. Ison JR. Temporal acuity in auditory function in the rat: Reflex inhibition by brief gaps in noise. J Comp Physiol Psychol. 1982;96(6):945-954.

41. Berger JI, Owen W, Wilson CA, Hockley A, Coomber B, Palmer AR, et al. Gap-induced reductions of evoked potentials in the auditory cortex: A possible objective marker for the presence of tinnitus in animals. Brain Res. 2018;1679:101-108. doi: 10.1016/j. brainres.2017.11.026.

42. Park SY, Kim MJ, Park JM, Park SN. A Mouse Model of Tinnitus Using Gap Prepulse Inhibition of the Acoustic Startle in an Accelerated Hearing Loss Strain. Otol Neurotol. 2020;41(4):e516-525. doi:10.1097/MAO.0000000000002573.

43. Gilani VM, Ruzbahani M, Mahdi P, Amali A, Khoshk MHN, Sameni J, et al. Temporal processing evaluation in tinnitus patients: Results on analysis of gap in noise and duration pattern test. Iran $J$ Otorhinolaryngol. 2013;25(73):221-225.

44. Boyen K, Ba kent D, Van Dijk P. The gap detection test: Can it be used to diagnose tinnitus? Ear Hear. 2015;36(4):e138-145. doi: 10.1097/ AUD.0000000000000156.

45. Campolo J, Lobarinas E, Salvi R. Does tinnitus «fill in» the silent gaps? Noise Heal. 2013;15(67):398-405. doi: 10.4103/1463-1741.121232.

46. Lee Y, López DE, Meloni EG, Davis M. A primary acoustic startle pathway: Obligatory role of cochlear root neurons and the nucleus reticularis pontis caudalis. J Neurosci. 1996;16(11):3775-3789. doi: 10.1523/JNEUROSCI.16-11-03775.1996.

47. Morse K, Vander Werff KR. Comparison of silent gap in noise cortical auditory evoked potentials in matched tinnitus and no-tinnitus control subjects. Am J Audiol. 2019;28(2):260-273. doi: 10.1044/2018_ AJA-18-0074. 